\title{
Validation of Integral Method for Numerical Prediction of Hybrid Rocket Internal Ballistics*
}

\author{
Yuki FUNAMI** and Toru SHIMADA*** \\ ** School of Engineering, The University of Tokyo \\ 7-3-1 Hongo, Bunkyo-ku, Tokyo 113-8656, Japan \\ E-mail: funami_yuki@ac.jaxa.jp \\ *** Institute of Space and Astronautical Science, Japan Aerospace Exploration Agency \\ 3-1-1 Yoshinodai, Chuo-ku, Sagamihara, Kanagawa 252-5210, Japan
}

\begin{abstract}
In order to design hybrid rocket engines, we developed a numerical prediction method which describes the internal ballistics, thus the solid fuel regression rate. In order to obtain a fast code, simple but efficient models have been implemented. The fluid dynamics and the thermal heat conduction in the solid fuel have longer characteristic times than chemical phenomena, hence time-dependency is required. The regression rate is evaluated by solving the energy-balance equation at the solid fuel surface. The code validation is made by comparing calculation results with open literature experimental data. This comparison confirmed that the numerical estimation of time- and space-averaged regression rates has the same order of magnitude as the experimental data of time- and space-averaged regression rates. However the dependency of regression rate on oxidizer mass flux differs between the calculations and the experiments. This difference could be mainly due to the use of only convective heat transfer between flame and fuel surface. Nevertheless, by considering also the radiative heat transfer, it is possible to obtain a higher accuracy in the calculation of the regression rate dependency on oxidizer mass flux.
\end{abstract}

Key words : Hybrid Rocket, Internal Ballistics, Numerical Prediction, Validation, Heat Feedback

\section{Introduction}

Hybrid rocket propulsion is considered a promising technology for the next generation of space transportation. A hybrid rocket typically features the fuel in the solid phase, while the oxidizer in the liquid or gas phase. If compared with solid rocket propulsion, hybrid technology is characterized by intrinsic safety and high theoretical specific impulse. Nevertheless, some technical drawbacks have to be overcome before developing hybrid rocket propulsion into practical space applications. Low fuel regression rate, low combustion efficiency and combustion instability are main disadvantages. As one can see in Fig. 1, the hybrid combustion mechanism is characterized by a diffusion flame within the turbulent boundary layer developed on the fuel surface. The gasification of the solid fuel is promoted by heat transfer from the flame, with convective and radiative mechanisms, to the fuel surface. The rate of gasification depends on many factors, such as convective and radiative heat transfer, blowing effect and heat conduction inside the solid fuel.

Many researchers developed the simulation codes for fuel regression rate prediction, such as Antoniou et al., University of New Orleans ${ }^{(1)}$. However, these simulation analyses consider short combustion times, less than about 10 seconds in two-dimensional cases while no more than 20 seconds in one- or zero-dimensional cases. Concerning combustion instability, for example, a specific simulation code was developed by Stoia-Djeska et al., Politehnica University of Bucharest ${ }^{(2)}$. Nevertheless, this code has been applied only to few cases and a

*Received 1 Feb., 2013 (No. 13-0059) [DOI: 10.1299/jfst.8.172]

Copyright (c) 2013 by JSME 


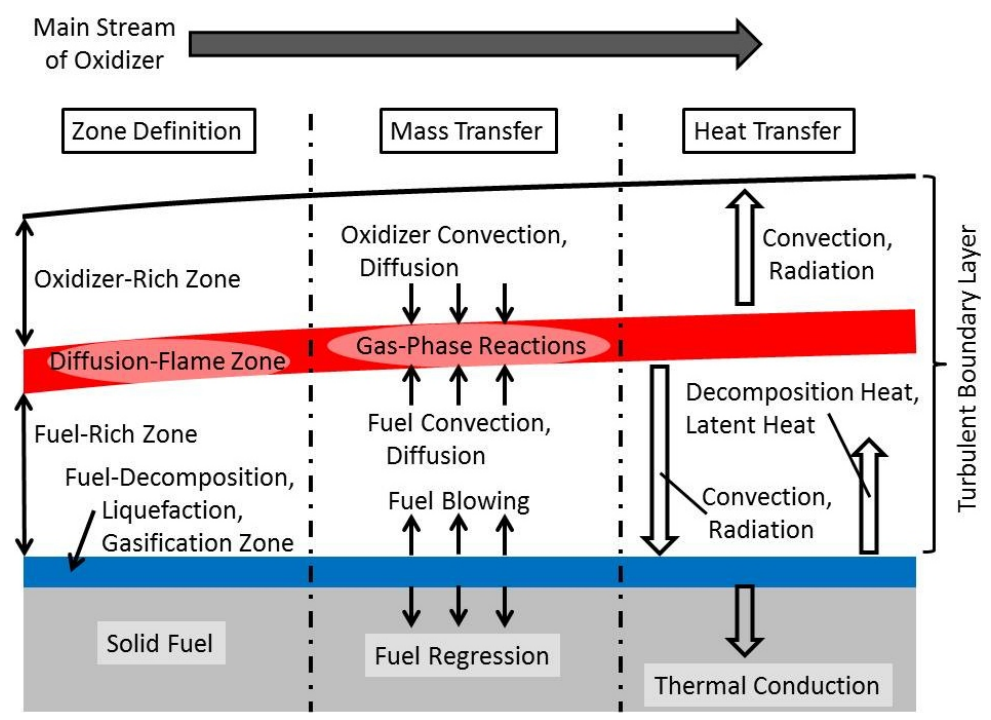

Fig. 1 Schematic of boundary layer combustion.

parametric study has not been conducted.

We have developed a design tool for hybrid rocket internal ballistics analysis, such as the estimation of fuel regression rates under various operating conditions, and parametric study. We did not consider a rigorous turbulent flame mathematical model (i.e. direct numerical simulation) because of its high calculation cost. Hence we have employed a faster model, which includes quasi-one-dimensional flowfield and one-dimensional thermal conduction into the solid fuel. In this model, the fluid dynamics and the thermal heat conduction into the solid fuel have longer characteristic times than chemical phenomena. On the other hand, characteristic times of combustion reactions are significantly short and quasi-steady flame can be assumed. In our previous study ${ }^{(3)}$, two combustion models were examined. One was the global single-step reaction model for 4 chemical species and the other one was the chemical equilibrium model for 9 chemical species. As a result, the former model was not appropriate to predict the state in the chamber, because the maximum temperature was enormously high. Therefore we use the latter model in this paper. In order to estimate regression rate, the energy-flux balance equation at the solid fuel surface is also solved.

The purpose of this paper is the validation of our method by comparing numerical results with experimental data available in the open literature. Furthermore, we discuss the dependency of fuel regression rate on oxidizer mass flux, considering the heat feedback from the flame to the solid surface.

\section{Nomenclature}

$A$ : cross-sectional area, $\mathrm{m}^{2}$

$A_{c}$ : preexponential factor, $1 / \mathrm{s}$

$B_{a}$ : aerodynamic blowing parameter, -

$B_{t}$ : thermochemical blowing parameter,

$C_{f} / C_{f 0}$ : ratio of skin-friction coefficient with/without surface blowing, -

$D_{p, 0}:$ diameter of initial port, $\mathrm{m}$

$D_{t}$ : diameter of nozzle throat, $\mathrm{m}$

$E_{a}:$ activation energy, $\mathrm{J} / \mathrm{mol}$

$F:$ Helmholtz free energy of mixture gas with constraints, $\mathrm{J} / \mathrm{kg}$

$G$ : total mass flux, $\mathrm{kg} /\left(\mathrm{m}^{2} \cdot \mathrm{s}\right)$
$G_{o x}$ : oxidizer mass flux, $\mathrm{kg} /\left(\mathrm{m}^{2} \cdot \mathrm{s}\right)$

$L_{p}:$ port length, $\mathrm{m}$

$N_{c}$ : number of cells, -

$Q_{c}$ : heat of combustion per mass unit of mixture gas, $\mathrm{J} / \mathrm{kg}$

$R_{e}$ : residual of specific total energy, $\mathrm{J} / \mathrm{kg}$

$R_{p}$ : residual of pressure, $\mathrm{Pa}$

$R_{u}$ : universal gas constant, $\mathrm{J} /(\mathrm{mol} \cdot \mathrm{K})$

$T$ : temperature, $\mathrm{K}$

$T_{0}$ : base temperature, $\mathrm{K}$

$Y_{i}$ : mass fraction of chemical species $i$, 
$a$ : constant parameter in Eq. (30), -

$b_{i}$ : mole number of element $i$ per mass unit of mixture gas, $\mathrm{mol} / \mathrm{kg}$

$c_{p, i}$ : specific heat of chemical species $i$ at constant pressure, $\mathrm{J} /(\mathrm{kg} \cdot \mathrm{K})$

$e:$ specific internal energy, $\mathrm{J} / \mathrm{kg}$

$e_{\text {reaction }}:$ degradation zone thickness, $\mathrm{m}$

$e_{t}:$ specific total energy, $\mathrm{J} / \mathrm{kg}$

$f$ : Helmholtz free energy of mixture gas, $\mathrm{J} / \mathrm{kg}$

$h_{i}$ : specific enthalpy of chemical species $i, \mathrm{~J} / \mathrm{kg}$

$h_{v}$ : effective heat of gasification of the solid fuel, $\mathrm{J} / \mathrm{kg}$

$h_{w}$ : enthalpy of the fuel gas evaporating from the solid fuel surface, $\mathrm{J} / \mathrm{kg}$

$k$ : constant parameter in Eqs. (9) and (16), -

$l_{p}$ : perimeter of a cross-section, $\mathrm{m}$

$\dot{m}_{F}$ : mass addition from the solid fuel surface, $\mathrm{kg} /\left(\mathrm{m}^{2} \cdot \mathrm{s}\right)$

$\dot{m}_{o x}$ : injection oxygen mass flow rate, $\mathrm{kg} / \mathrm{s}$

$n:$ constant parameter in Eq. (30), -

$p:$ pressure, $\mathrm{Pa}$

$q$ : constant parameter in Eqs. (9) and (16), -

$\dot{q}_{R}$ : radiative energy flux, $\mathrm{W} / \mathrm{m}^{2}$

Subscripts

1 : fuel stream

2 : oxidizer stream

$F$ : fuel

$P$ : combustion products

$S:$ solid fuel surface

$a$ : ambient

$e:$ boundary layer edge

\section{Superscripts}

- : time- and/or space-average

0 : constraint condition $r:$ port radius, $\mathrm{m}$

$\dot{r}:$ fuel regression rate, $\mathrm{m} / \mathrm{s}$

$t:$ time, $\mathrm{s}$

$t_{b}:$ burning period, $\mathrm{s}$

$u: x$-direction velocity, $\mathrm{m} / \mathrm{s}$

$v: y$-direction velocity, $\mathrm{m} / \mathrm{s}$

$w_{i}$ : molecular weight of chemical species $i, \mathrm{~kg} / \mathrm{mol}$

$x$ : axial location from head-end of the chamber, $\mathrm{m}$

$y$ : location normal to the solid fuel surface, $\mathrm{m}$

$\Delta h_{i}^{0}$ : standard enthalpy of formation of chemical species $i, \mathrm{~J} / \mathrm{kg}$

$\Delta m_{F}$ : fuel mass loss, $\mathrm{kg}$

$\Delta t:$ time interval, $\mathrm{s}$

$\Lambda$ : Lagrangian multiplier, $\mathrm{J} / \mathrm{mol}$

$\alpha:$ thermal diffusivity, $\mathrm{m}^{2} / \mathrm{s}$

$\alpha_{R}:$ absorptivity, -

$\varepsilon$ : emissivity, -

$\lambda:$ thermal conductivity, $\mathrm{W} /(\mathrm{m} \cdot \mathrm{K})$

$\mu$ : viscosity, $\mathrm{Pa} \cdot \mathrm{s}$

$\xi:$ mixture fraction, -

$\pi$ : circle ratio, -

$\rho:$ density, $\mathrm{m}^{3}$

$\sigma$ : Stefan-Boltzmann constant, $\mathrm{W} /\left(\mathrm{m}^{2} \cdot \mathrm{K}^{4}\right)$

final : final state

$f l$ : flame location

$g:$ gas

initial : initial state

$r:$ reference

$s:$ solid

$n$ : time step

\section{Mathematical Models and Numerical Methods}

\subsection{Configuration of the Hybrid Rocket Engine}

A typical hybrid rocket engine is composed of the combustion chamber, which contains the solid fuel, the de Laval nozzle and the feeding system for the oxidizer (pressurization system, tank and injector). The fuel grain, considered in this paper, has a single circular central port. The oxidizer flow is axially injected into the chamber. In this simulation, the one-dimensional calculation domain corresponding to the axial direction involves only the combustion chamber and the nozzle as one can see in Fig. 2. The calculation domain in the chamber is divided into 300 cells while the domain in the nozzle is divided into 200 cells.

The solid fuel is high density polyethylene (HDPE) and the oxidizer is gaseous oxygen (GOX). It is assumed that, during gasification processes, each polyethylene chain is broken 


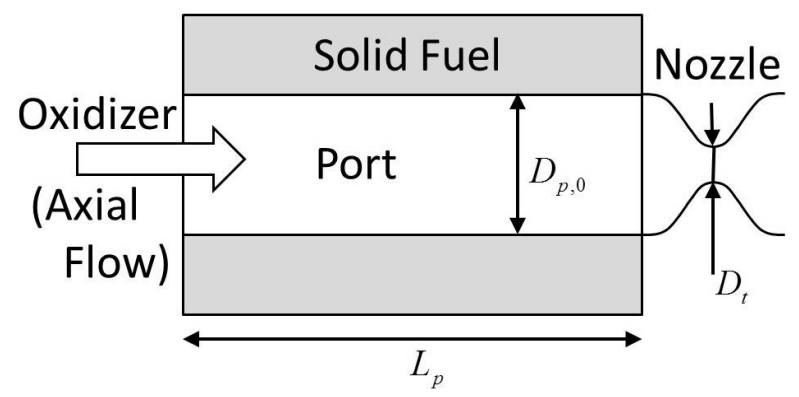

Fig. 2 Calculation domain.

into ethylene molecules.

\subsection{Quasi-One-Dimensional Flowfield}

For simplicity, quasi-one-dimensional flowfield is assumed. The representative values of physical quantities are area-mean values, and their changes along the axial direction are considered. The flowfield is described by the compressible Euler equations with a conservation equation for the mixture fraction. The mass addition from the solid fuel surface is introduced as a source term.

$$
\begin{aligned}
& \frac{\partial A \mathbf{Q}}{\partial t}+\frac{\partial A \mathbf{F}}{\partial x}=\mathbf{S}_{Q 1 D}+\mathbf{S}_{\text {Mass }}, \\
& \mathbf{Q}=\left(\begin{array}{c}
\rho \\
\rho u \\
\rho e_{t} \\
\rho \xi
\end{array}\right), \mathbf{F}=\left(\begin{array}{c}
\rho u \\
\rho u^{2}+p \\
\left(\rho e_{t}+p\right) u \\
\rho \xi u
\end{array}\right), \mathbf{S}_{Q 1 D}=\left(\begin{array}{c}
0 \\
p \frac{d A}{d x} \\
0 \\
0
\end{array}\right), \mathbf{S}_{\text {Mass }}=\left(\begin{array}{c}
l_{p} \dot{m}_{F} \\
0 \\
l_{p} \dot{m}_{F} h_{w} \\
l_{p} \dot{m}_{F}
\end{array}\right) .
\end{aligned}
$$

These equations are solved numerically. Boundary conditions at head-end of the chamber are subsonic inflow conditions as shown in Table 1. At the nozzle exit, supersonic outflow conditions are imposed.

Mixture fraction $\xi$ is defined as follows,

$$
\xi \equiv \frac{b_{\mathrm{C}}-b_{\mathrm{C}, 2}}{b_{\mathrm{C}, 1}-b_{\mathrm{C}, 2}}
$$

where $b_{C}$ is mole number of element $\mathrm{C}$ per mass unit of mixture gas. This parameter presents the ratio between fuel and oxidizer. It is related to temperature and chemical composition of combustion gas with chemical equilibrium calculation (see $\$ 2.3$ ).

Specific total energy of mixture gas $e_{t}$ is expressed as follows,

$$
\begin{aligned}
& e_{t}=e+\frac{u^{2}}{2}, \\
& e=\sum_{i} h_{i} Y_{i}-\frac{p}{\rho}, \\
& h_{i}=\Delta h_{i}^{0}+\int_{T_{r}}^{T} c_{p, i} d T .
\end{aligned}
$$

Specific heat at constant pressure $c_{p, i}$ is a function of temperature. The function is obtained by fitting the JANAF table's data ${ }^{(4)}$.

The equation of state for ideal gas is

$$
p=\sum_{i} \frac{Y_{i}}{w_{i}} \rho R_{u} T \text {. }
$$

When Eq. (1) is solved, mass addition $\dot{m}_{F}$ must be obtained in advance, by solving the equation of energy-flux balance at the solid fuel surface (see $\$ 2.4$ ).

Table 1 Inflow conditions.

\begin{tabular}{cccc}
\hline Mass flow rate & Pressure & Temperature & Mixture fraction \\
\hline given & zeroth-order extrapolation & $300[\mathrm{~K}]$ & $0[-]$
\end{tabular}




\subsection{Chemical Equilibrium}

Many chemical species take part in the combustion reactions and a lot of elementary processes are involved at the same time. However when a combustion calculation step is completed, it is difficult that all chemical species and all elementary processes are considered. Hence the combustion model should deal with only significant chemical species and elementary processes during the combustion. Because of this consideration, a faster code is suitable using the chemical equilibrium model for 9 chemical species. With NASA CEA $\operatorname{code}^{(4)}$, chemical species are selected so that the flame temperature in limitation of chemical species is nearly equal to that in no limitation of chemical species. As the result, 9 chemical species $\mathrm{C}_{2} \mathrm{H}_{4}, \mathrm{O}_{2}, \mathrm{CO}_{2}, \mathrm{H}_{2} \mathrm{O}, \mathrm{CO}, \mathrm{H}_{2}, \mathrm{OH}, \mathrm{O}$ and $\mathrm{H}$ are considered. Particular reaction processes are not considered.

It is assumed that gasified fuel and oxidizer are mixed and reacted instantly in the control volume. Temperature and chemical composition of the combustion gas are obtained with chemical equilibrium calculation. In this calculation, free energy is minimized by Lagrange's method of undetermined multipliers under the constraint condition that mole numbers of elements are conserved ${ }^{(4)}$. Helmholtz free energy is used for the free energy minimization, and the values of density $\rho$, internal energy $e$ and mixture fraction $\xi$ are required. Minimized function $F$ is given as follows,

$$
F=f+\sum_{i} \Lambda_{i}\left(b_{i}-b_{i}^{0}\right) .
$$

\subsection{Energy-Flux Balance at the Solid Fuel Surface}

In order to estimate fuel regression rate $\dot{r}$, i.e. fuel mass addition $\dot{m}_{F}\left(=\rho_{s} \dot{r}\right)$, energy-flux balance at the solid fuel surface is implemented, which is described as follows. In this section, radiative heat transfer is not considered. The case including radiation will be discussed later (see §3.4).

$$
\lambda_{g}\left(\frac{\partial T}{\partial y}\right)_{y=+0}=\lambda_{s}\left(\frac{\partial T}{\partial y}\right)_{y=-0}+\rho_{s} \dot{r} h_{v} .
$$

Coordinate $y$ is normal to the solid fuel surface. The origin of this coordinate system is fixed on the solid fuel surface. Negative values of $y$ are referred to the inside part of the fuel. It is assumed that the solid fuel is semi-infinite, i.e. $-\infty<y \leq 0$. Considering Eq. (8), the lefthand side is the heat feedback from the flam, the first term in the right-hand side is the thermal conduction into the solid fuel, while the second term is the energy required for the solid fuel to pyrolyze and to gasify.

The left-hand side of Eq. (8) is evaluated by quasi-steady flame model, such as Karabeyoglu's model ${ }^{(5)}$. Karabeyoglu's model is based on Marxman's turbulent boundary layer combustion model ${ }^{(6)}$. While thermochemical blowing parameter is equal to aerodynamic blowing parameter in Marxman's model, Karabeyoglu distinguishes these parameters.

$$
\lambda_{g}\left(\frac{\partial T}{\partial y}\right)_{y=+0}=\left[\frac{0.03 q}{\mu^{-0.2}}\right]^{\frac{1}{1-k}}\left[\frac{B_{t} h_{v}}{\rho_{s}^{k /(1-k)}}\right] x^{-0.2 /(1-k)} G^{0.8 /(1-k)} \dot{r}^{-k /(1-k)} .
$$

This equation, Eq. (9), expresses the convective heat transfer in the turbulent boundary layer. One can see that the heat feedback behavior depends on total mass flux $G$.

The thermochemical blowing parameter in Eq. (9) is represented by $B_{t}$. This parameter affects the heat transfer in boundary layer. It is defined as follows,

$$
B_{t} \equiv \frac{u_{e}}{u_{f l}} \frac{h_{f l}-h_{S}}{h_{v}} .
$$

The specific enthalpy at the flame $h_{f l}$ and the specific enthalpy at the surface $h_{S}$ are defined with base temperature $T_{0}{ }^{(7)}$. It is assumed that the gas composition at the surface is $100 \%$ 
Table 2 Parameters related to the $B_{t}$ evaluation.

\begin{tabular}{ll}
\hline Parameter & Value [Unit] \\
\hline Base temperature $T_{0}$ & $298.15[\mathrm{~K}]$ \\
Heat of combustion per mass unit of mixture gas $Q_{c}$ & $5300[\mathrm{~kJ} / \mathrm{kg}]$ \\
Effective heat of gasification $h_{v}{ }^{\left({ }^{2}\right)}$ & $2700[\mathrm{~kJ} / \mathrm{kg}]$ \\
Velocity ratio $u_{e} / u_{f l}{ }^{(7)}$ & $1.8[-]$ \\
\hline
\end{tabular}

fuel.

$$
\begin{aligned}
& h_{f l}=h_{P}\left(T_{f l}\right)-h_{P}\left(T_{0}\right), \\
& h_{S}=h_{F}\left(T_{S}\right)-h_{F}\left(T_{0}\right) .
\end{aligned}
$$

Assuming adiabatic conditions, the specific enthalpy at the flame $h_{f l}$ is equal to the heat of combustion per mass unit of mixture gas $Q_{c}$,

$$
h_{f l}=Q_{c} .
$$

While the specific enthalpy at the surface $h_{S}$ was constant in our previous study ${ }^{(3)}$, it is a function of surface temperature $T_{S}$ in this paper. The function is obtained by fitting the JANAF table's data ${ }^{(4)}$. The parameter list is shown in Table 2.

The constant parameters $q, k$ in Eq. (9) are related to skin-friction coefficient. The ratio of skin-friction coefficient with/without surface blowing $C_{f} / C_{f 0}$ means the reduction in skinfriction caused by surface blowing, which is called "blocking effect". Marxman expressed the factor $C_{f} / C_{f 0}$ as a function of aerodynamic blowing parameter $B_{a}{ }^{(6)}$.

$$
\begin{aligned}
& \frac{C_{f}}{C_{f 0}}=\left[\frac{\ln \left(1+B_{a}\right)}{B_{a}}\right]^{0.8}\left[\frac{1+1.3 B_{a}+0.364 B_{a}^{2}}{\left(1+0.5 B_{a}\right)^{2}\left(1+B_{a}\right)}\right]^{0.2}, \\
& B_{a} \equiv \frac{(\rho v)_{S}}{\rho_{e} u_{e} C_{f} / 2}=\frac{\rho_{s} \dot{r}}{G C_{f} / 2} .
\end{aligned}
$$

He also suggested the simplified expression in a particular range of $B_{a}$.

$$
\frac{C_{f}}{C_{f 0}}=q B_{a}^{-k} .
$$

The parameters $q, k$ in Eq. (9) are defined by Eq (16). In this paper, the range $1 \leq B_{a} \leq 15$ is considered. When Eq. (14) is applied to this range of $B_{a}, q=0.74$ and $k=0.53$ are obtained.

In the first term of the Eq. (8) right-hand side, temperature profile in the solid fuel is obtained by solving one-dimensional thermal conduction equation at any location $x$,

$$
\frac{\partial T}{\partial t}+\dot{r} \frac{\partial T}{\partial y}-\alpha_{s} \frac{\partial^{2} T}{\partial y^{2}}=0
$$

with following boundary conditions

$$
\begin{aligned}
& T=T_{S} \quad \text { at } y=0, \\
& T=T_{a} \text { at } y \rightarrow-\infty .
\end{aligned}
$$

In a steady state, analytical solution of this equation can be obtained,

$$
T(y)=T_{a}+\left(T_{S}-T_{a}\right) \exp \left(\frac{\dot{r}}{\alpha_{s}} y\right) .
$$

Hence the heat flux into the solid fuel is given as follows,

$$
\lambda_{s}\left(\frac{\partial T}{\partial y}\right)_{y=-0}=\lambda_{s}\left(T_{S}-T_{a}\right) \frac{\dot{r}}{\alpha_{s}} .
$$

In a hybrid rocket, fuel regression is mainly caused by fuel pyrolysis and gasification. Arrhenius-type equation is empirically used as pyrolysis rate equation ${ }^{(8)}$.

$$
\dot{r}=A_{c} \exp \left(-\frac{E_{a}}{R_{u} T_{S}}\right) e_{\text {reaction }} .
$$


Table 3 Parameters in Arrhenius-type equation ${ }^{(8)}$.

\begin{tabular}{ll}
\hline Parameter & Value [Unit] \\
\hline Preexponential factor $A_{c}$ & $2.0 \times 10^{16}[1 / \mathrm{s}]$ \\
Activation energy $E_{a}$ & $1.5 \times 10^{5}[\mathrm{~J} / \mathrm{mol}]$ \\
Degradation zone thickness $e_{\text {reaction }}$ & $25[\mu \mathrm{m}]$ \\
\hline
\end{tabular}

The parameter list is shown in Table 3. Regression rate $\dot{r}$ is eliminated from Eq. (8) by using Eq. (22). Hence, Eq. (8) is expressed as the equation of temperature at the solid fuel surface $T_{S}$. By solving Eq. (8) for $T_{S}$, it is possible to evaluate $\dot{r}$ and $\dot{m}_{F}$.

\subsection{Numerical Schemes}

Equation (1) is discretized with finite volume method. Numerical flux is evaluated with AUSMDV scheme which is famous for its robustness ${ }^{(9)}$. In order to achieve higher order accuracy for space, second order MUSCL method is used. Time integration is two-stage Runge-Kutta method.

It is assumed that surface regression is a quasi-static change. At a certain time $t$, the convergent result of Eq. (1) is calculated. The convergence conditions and the definitions of residuals are described as follow,

$$
\begin{aligned}
& R_{e} \leq 1.0 \times 10^{-4} \text { and } R_{p} \leq 1.0 \times 10^{-4}, \\
& R_{e} \equiv \frac{\sqrt{\sum_{i=1}^{N_{c}}\left(e_{t, i}^{n+1}-e_{t, i}^{n}\right)^{2}}}{N_{c}} \\
& R_{p} \equiv \frac{\sqrt{\sum_{i=1}^{N_{c}}\left(p_{i}^{n+1}-p_{i}^{n}\right)^{2}}}{N_{c}}
\end{aligned}
$$

Port configuration at $t+\Delta t$ (where $\Delta t=0.3[\mathrm{~s}]$ ) is evaluated with the obtained regression rate, and the convergent result is calculated again. This procedure is iterated during the burning period $t_{b}$. Time-histories of the state in the chamber can be obtained by compiling the convergent results.

\section{Results and Discussion}

\subsection{Comparison between Calculations and Experimental Data for Averaged Regression} Rate

In order to validate our method, calculation results are compared with experimental data available in the open literature. The conditions of the calculations are conformed to Karebeyoglu's experimental conditions ${ }^{(10)}$ as shown in Table 4.

In Fig. 3 (a), one can see the pressure time-history for Test No.1 which is a typical case in calculation results. Chamber pressure decreases gradually with time. Local regression rate profiles for Test No.1 (see Fig. 3 (b)) is maximum at the head-end of the chamber, decreasing gradually with time.

In Fig. 4, time- and space-averaged numerical regression rates $\bar{r}$ are compared with data obtained by the experiments. The averaging method of numerical results is described follow,

$$
\Delta m_{F}=\rho_{s} \int_{0}^{L_{p}} \pi\left(r_{\text {final }}^{2}-r_{\text {initial }}^{2}\right) d x,
$$

Table 4 Experimental conditions ${ }^{(10)}$.

\begin{tabular}{cccccc}
\hline Test number & $\begin{array}{c}\text { Port length, } \\
L_{p}[\mathrm{~cm}]\end{array}$ & $\begin{array}{c}\text { Diameter of } \\
\text { initial port, } \\
D_{p, 0}[\mathrm{~mm}]\end{array}$ & $\begin{array}{c}\text { Diameter of } \\
\text { nozzle throat, } \\
D_{t}[\mathrm{~mm}]\end{array}$ & $\begin{array}{c}\text { Injection oxygen } \\
\text { mass flow rate, } \\
\dot{m}_{o x}[\mathrm{~kg} / \mathrm{s}]\end{array}$ & $\begin{array}{c}\text { Burning period, } \\
t_{b}[\mathrm{~s}]\end{array}$ \\
\hline 1 & 30.48 & 12.8 & 9.78 & 0.0454 & 3.0 \\
2 & 30.48 & 17.0 & 9.78 & 0.0453 & 3.0 \\
3 & 30.48 & 22.0 & 9.53 & 0.0328 & 3.0 \\
4 & 30.48 & 12.8 & 9.91 & 0.0329 & 3.0 \\
\hline
\end{tabular}




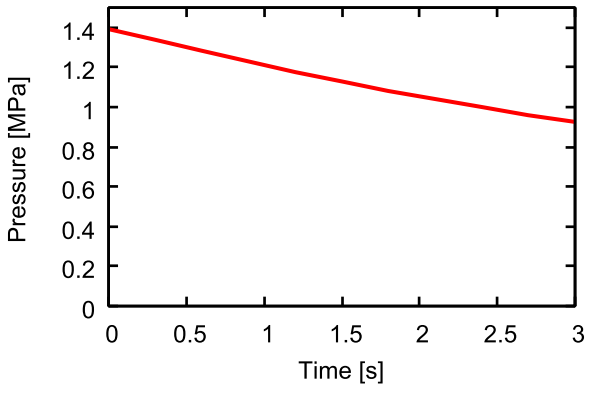

(a) Pressure time-history near the port-end.

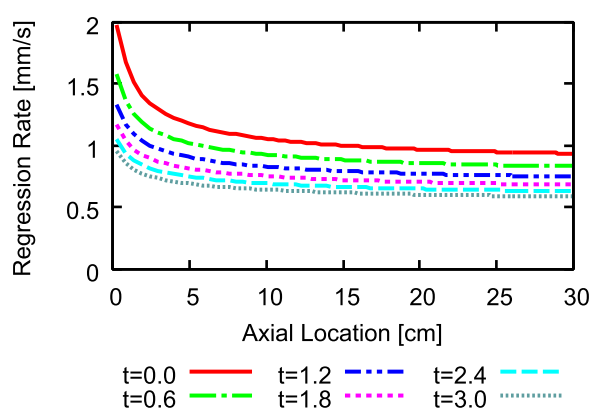

(b) Local regression rate profiles. Unit of $t$ is second.

Fig. 3 Typical calculation result (Test No.1).

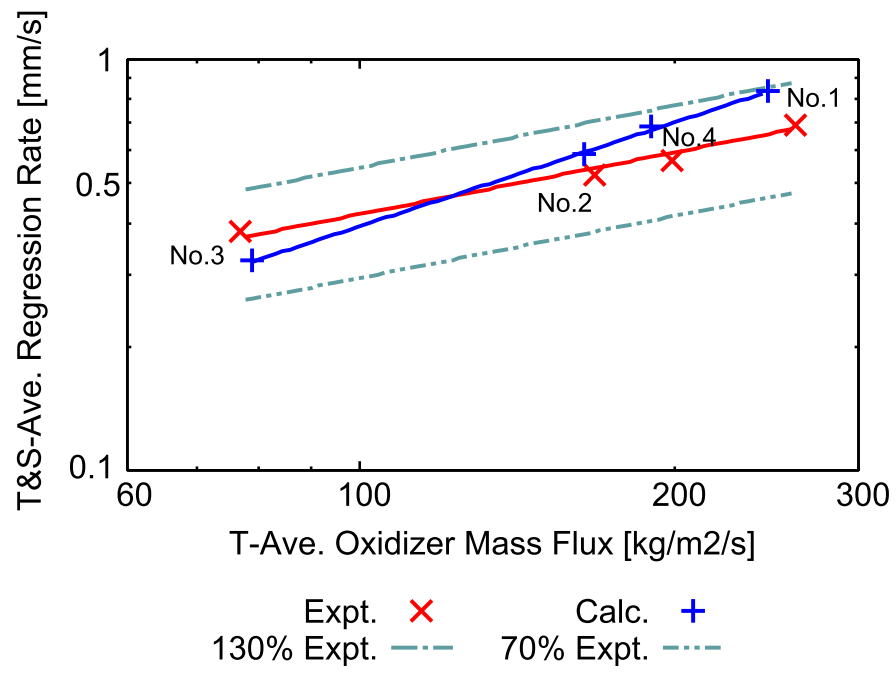

Fig. 4 Time-averaged oxidizer mass flux .vs. Time- and space-averaged regression rate. The interpolated lines are the fitted lines to the empirical expression $\bar{r}=a \bar{G}_{o x}^{n}$. The $x$-axis and the $y$-axis are $\log$ scale.

$$
\begin{aligned}
& \bar{r}_{\text {final }}=\sqrt{r_{\text {initial }}^{2}+\frac{\Delta m_{F}}{\pi \rho_{s} L_{p}}}, \\
& \bar{r}=\frac{\bar{r}_{\text {final }}-r_{\text {initial }}}{t_{b}}, \\
& \bar{G}_{o x}=\frac{4 \dot{m}_{o x}}{\pi\left(r_{\text {initial }}+\bar{r}_{\text {final }}\right)^{2}} .
\end{aligned}
$$

The values of numerical $\overline{\dot{r}}$ fall within $\pm 30 \%$ range of the experimental data fitting. One can see that the order of magnitude of averaged numerical regression rate is the same of the experimental data.

The calculation results and the experimental data are fitted in the empirical expression as follows,

$$
\bar{r}=a \bar{G}_{o x}^{n} .
$$

In Eq. (30), unit of $\overline{\dot{r}}$ is $\mathrm{mm} / \mathrm{s}$ and unit of $\bar{G}_{o x}$ is $\mathrm{kg} /\left(\mathrm{m}^{2} \cdot \mathrm{s}\right)$. The constant parameters $a$ and $n$ are determined by least-square method, resulting in $(a, n)=(0.00821,0.841)$ for the calculations and $(a, n)=(0.0421,0.498)$ for the experiments. The factor $n$ represents the regression rate dependency on oxidizer mass flux. This factor in the calculations differs from that in the experiments. In fact, in this case the $n$ value is higher. However, from statistical point of view, only four data points are not enough for an accurate estimation of the factors $a$ and $n$. For example, Karabeyoglu obtained the factor $n(=0.62)$ in the GOX/paraffin system by using 
Table 5 The factors $a$ and $n$ in the various values of activation energy $E_{a}$.

\begin{tabular}{cccccc}
\hline & $1.5 E_{a, r}$ & $1.1 E_{a, r}$ & $1.0 E_{a, r}$ & $0.9 E_{a, r}$ & $0.5 E_{a, r}$ \\
\hline $\mathrm{a}$ & 0.00667 & 0.00801 & 0.00821 & 0.00864 & 0.00960 \\
$\mathrm{n}$ & 0.815 & 0.835 & 0.841 & 0.842 & 0.858 \\
\hline
\end{tabular}

much more than ten data points ${ }^{(11)}$. The improvement of this point will be performed in the future work.

\subsection{The Effect of Parameters $A_{c}$ and $E_{a}$ in the Arrhenius-Type Equation}

The Arrhenius-type equation, Eq. (22), is directly used by evaluating local regression rate $\dot{r}$. Hence it is inferred that the parameters $A_{c}$ and $E_{a}$ in this equation affect the regression rate significantly. In order to confirm this inference, the calculations with various values of $A_{c}$ and $E_{a}$ have been performed. At first, the value of activation energy $E_{a}$ is changed parametrically. The value in Table 3 sets a reference value $E_{a, r}$, and the values $1.5 E_{a, r}, 1.1 E_{a, r}, 1.0 E_{a, r}$, $0.9 E_{a, r}$ and $0.5 E_{a, r}$ are used by calculations. These results are shown in Table 5 and Fig. 5 . In this range of activation energy, the factor $n$ increases with decreasing activation energy and does not change greatly from about 0.8. On the other hand, the factor $a$ also increases with decreasing activation energy. Second, the value of preexponential factor $A_{c}$ is changed parametrically. The value in Table 3 sets a reference value $A_{c, r}$, and the values $100 A_{c, r}, 10 A_{c, r}$, $1.5 A_{c, r}, 1.1 A_{c, r}, 1.0 A_{c, r}, 0.9 A_{c, r}, 0.5 A_{c, r}, 0.1 A_{c, r}$ and $0.01 A_{c, r}$ are used by calculations. The calculation results are fitted into Eq. (30). The obtained factors $a$ and $n$ are shown in Fig. 6. In this range of preexponential factor, the factors $a$ and $n$ tend to increase with the increase of the preexponential factor. However, the variation ranges of $a$ and $n$ in the cases with changing $A_{c}$ are much more narrow than those in the cases with changing $E_{a}$. The activation energy $E_{a}$ affects the evaluation of the factors $a$ and $n$ much more than the preexponential factor $A_{c}$. In fact $E_{a}$ is in the exponent function of Eq. (22), unlike $A_{c}$.

\subsection{The Effect of Index Number of Mass Flux $G$ in the Heat Feedback Model}

In this section, we discuss how the index number of mass flux $G$ in the heat feedback model, Eq. (9), affects on the factor $n$ in the empirical expression, Eq. (30).

In the energy-flux balance equation at the solid fuel surface, Eq. (8), it is assumed that the heat flux of thermal conduction into the solid fuel is much smaller than the energy required

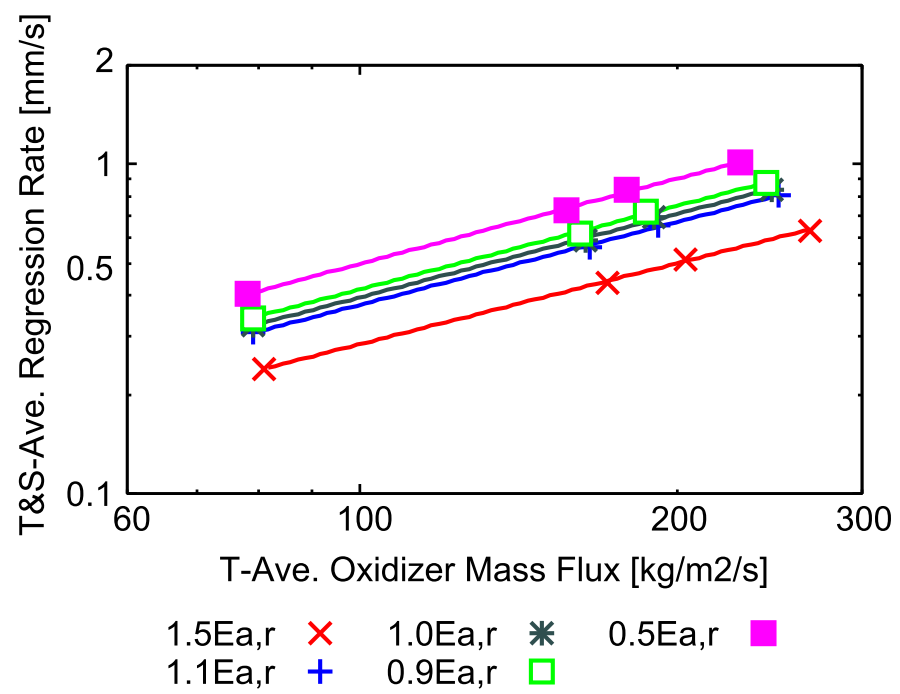

Fig. 5 Time- and space-averaged regression rate. The activation energy $E_{a}$ is changed into various values. The interpolated lines are the fitted lines to the empirical expression $\overline{\dot{r}}=a \bar{G}_{o x}^{n}$. The $x$-axis and the $y$-axis are log scale. 


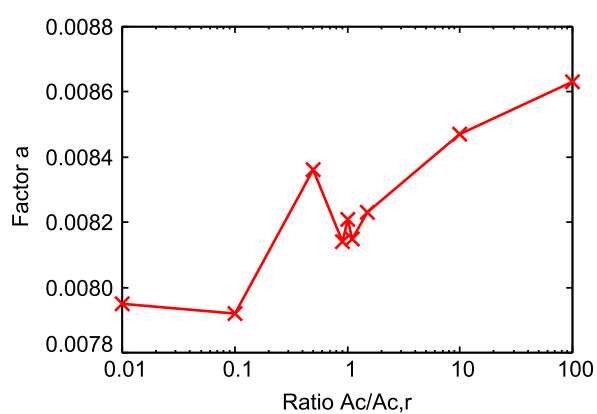

(a) Factor $a$

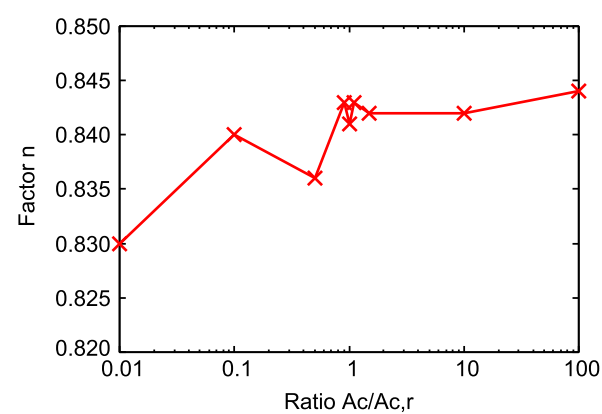

(b) Factor $n$.

Fig. 6 The values of the factors $a$ and $n$ of the empirical expression $\bar{r}=a \bar{G}_{o x}^{n}$. The preexponential factor $A_{c}$ is changed into various values. The $x$-axis is $\log$ scale.

for the solid fuel to pyrolyze and to gasify.

$$
\lambda_{s}\left(\frac{\partial T}{\partial y}\right)_{y=-0} \ll \rho_{s} \dot{r} h_{v}
$$

Actually, in the calculations, $\rho_{s} \dot{r} h_{v}$ is 3 to 4 times higher than $\lambda_{s}(\partial T / \partial y)_{y=-0}$. Under this assumption, the energy-flux balance equation can be simplified as follows,

$$
\lambda_{g}\left(\frac{\partial T}{\partial y}\right)_{y=+0} \approx \rho_{s} \dot{r} h_{v}
$$

The following equation, Eq. (33), is obtained with Eqs. (32) and (9),

$$
\dot{r} \propto G^{0.8} .
$$

Hence the dependency of local regression rate $\dot{r}$ on total mass flux $G$ is roughly determined by the index number of $G$ in Eq. (9).

It is inferred that the averaged regression rate $\overline{\dot{r}}$ also depends on the index number of $G$ in Eq. (9). This is confirmed by calculating $\overline{\dot{r}}$ after changing $G^{0.8 /(1-k)}$ into $G^{0.5 /(1-k)}$ in Eq. (9) (This model in which the index number of $G$ is changed into $0.5 /(1-k)$ is called "changed model"). In Fig. 7, one can see this calculation results, which, fitted in Eq. (30), provide $(a, n)=(0.00871,0.497)$. The value of $n$ in this calculations is almost the same as 0.5 of the index number of $G$ in the changed model. This confirms that the factor $n$ in Eq. (30) is greatly affected by the numerator of the index number of $G$ in Eq. (9). Next, the effect of the factor $k$, which can be eliminated with the assumption of Eq. (31), is examined. The value of the factor $k$ is changed parametrically by setting the reference value $k_{r}$ equal to 0.53 . The values $1.5 k_{r}, 1.1 k_{r}, 1.0 k_{r}, 0.9 k_{r}$ and $0.5 k_{r}$ are used by calculations. The calculation results are shown in Table 6 and Fig. 8. In this range of the factor $k$, the variation of the factor $n$ is very small.

The value 0.8 , of the index number of $G$ in the heat feedback model, means that the dominant form of the heat feedback from the flame to the fuel surface is the convective heat transfer in the turbulent boundary layer. In order to conform the estimated values to the experimental values of $n$ and $\overline{\dot{r}}$, a heat feedback model must include not only convective heat transfer but also other phenomena, such as radiative heat transfer, chamber pressure effect (i.e. finite-rate chemical reactions) and so on.

Table 6 The factors $a$ and $n$ in the various values of the factor $k$.

\begin{tabular}{cccccc}
\hline & $1.5 k_{r}$ & $1.1 k_{r}$ & $1.0 k_{r}$ & $0.9 k_{r}$ & $0.5 k_{r}$ \\
\hline $\mathrm{a}$ & 0.00679 & 0.00804 & 0.00821 & 0.00868 & 0.0101 \\
$\mathrm{n}$ & 0.837 & 0.837 & 0.841 & 0.838 & 0.842 \\
\hline
\end{tabular}




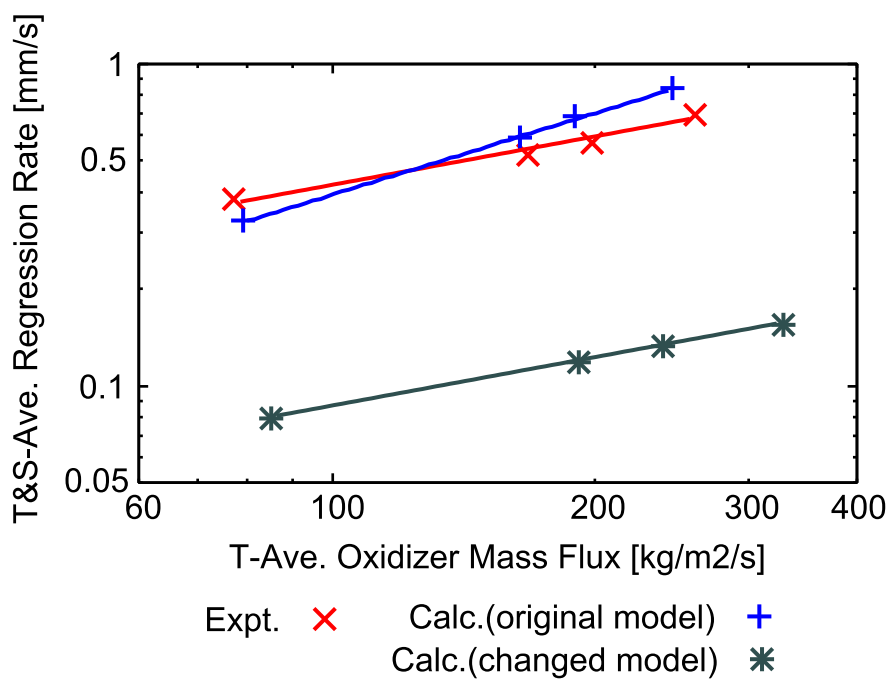

Fig. 7 Time- and space-averaged regression rates are estimated with the heat feedback model in which the index number of $G$ is changed into $0.5 /(1-k)$. The interpolated lines are the fitted lines to the empirical expression $\bar{r}=a \bar{G}_{o x}^{n}$. The $x$-axis and the $y$-axis are log scale.

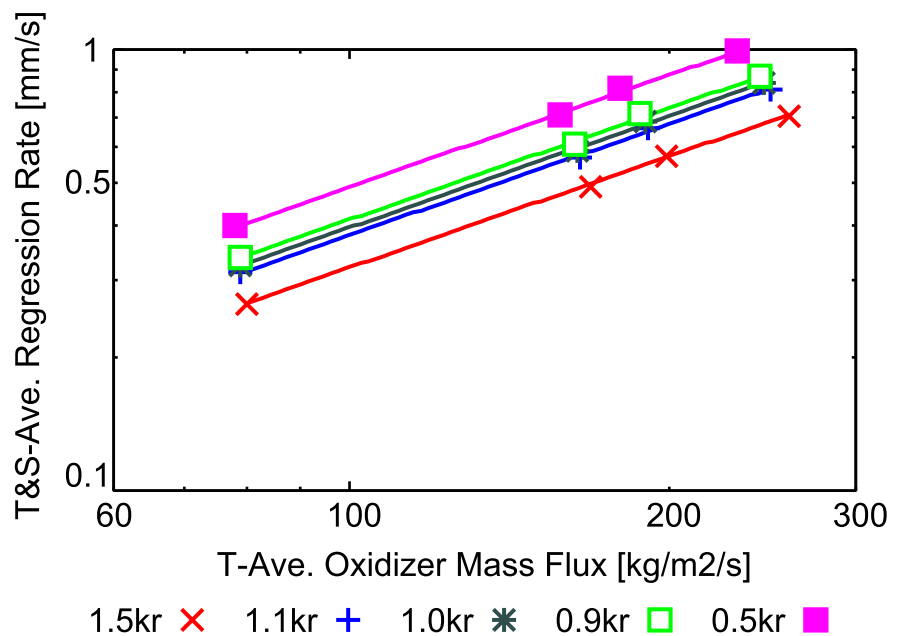

Fig. 8 Time- and space-averaged regression rate. The factor $k$ is changed into various values. The interpolated lines are the fitted lines to the empirical expression $\overline{\dot{r}}=a \bar{G}_{o x}^{n}$. The $x$-axis and the $y$-axis are log scale.

\subsection{The Effect of Radiative Heat Transfer}

As a first step, radiative heat transfer, which is one of the unconsidered phenomena in above sections, is now considered. For simplicity, it is assumed that all amount of energy transferred by radiation is absorbed at the solid fuel surface. The term of radiative heat transfer $\dot{q}_{R}$ is added into the energy-flux balance equation at the solid fuel surface, Eq. (8).

$$
\begin{aligned}
& \lambda_{g}\left(\frac{\partial T}{\partial y}\right)_{y=+0}+\dot{q}_{R}=\lambda_{s}\left(\frac{\partial T}{\partial y}\right)_{y=-0}+\rho_{s} \dot{r} h_{v}, \\
& \dot{q}_{R}=\alpha_{R, s} \varepsilon_{g} \sigma T_{f l}^{4}-\varepsilon_{s} \sigma T_{S}^{4} .
\end{aligned}
$$

The term $\dot{q}_{R}$ includes not only the energy transferred from the flame to the surface but also the energy transferred from the surface to the gas-phase region. The parametric calculations with radiation effects have been performed. The parameters for radiation $\varepsilon_{g}, \varepsilon_{s}$ and $\alpha_{R, s}$ are arbitrarily changed into various values as shown in Table 7. Here, Kirchhoff's law $\varepsilon_{s}=\alpha_{R, s}$ is assumed. The calculation results are shown in Figs. 9 and 10. The parameters $a$ and $n$ obtained by the results fitting in Eq. (30) are also listed in Table 7. In this range of emissivity, the factor $n$ increases with decreasing gas emissivity. On the other hand, the factor $n$ also 
Table 7 The parameters for radiation, the factors $a$ and $n$.

\begin{tabular}{c|cc|cc}
\hline Case number & Gas emissivity $\varepsilon_{g}$ & Solid fuel emissivity $\varepsilon_{s}$ & Factor $a$ & Factor $n$ \\
\hline 1 & 0 & 0 & 0.00821 & 0.841 \\
2 & 0.5 & 0.95 & 0.537 & 0.218 \\
3 & 0.3 & 0.95 & 0.273 & 0.284 \\
4 & 0.1 & 0.95 & 0.0327 & 0.613 \\
5 & 0.1 & 0.85 & 0.0284 & 0.636 \\
6 & 0.1 & 0.75 & 0.0245 & 0.660 \\
\hline
\end{tabular}

increases with the decreasing of solid fuel emissivity. The variation range of the factor $n$ in this calculation includes the value, 0.50 , obtained by the experimental data fitting. Hence the model with radiation allows to improve the prediction accuracy of the regression rate dependency on oxidizer mass flux, i.e. the factor $n$.

The next calculation should employ an estimation model of the parameters $\varepsilon_{g}, \varepsilon_{s}$ and $\alpha_{R, s}$ in the GOX/HDPE combustion system. Then the importance of the radiation effect should be

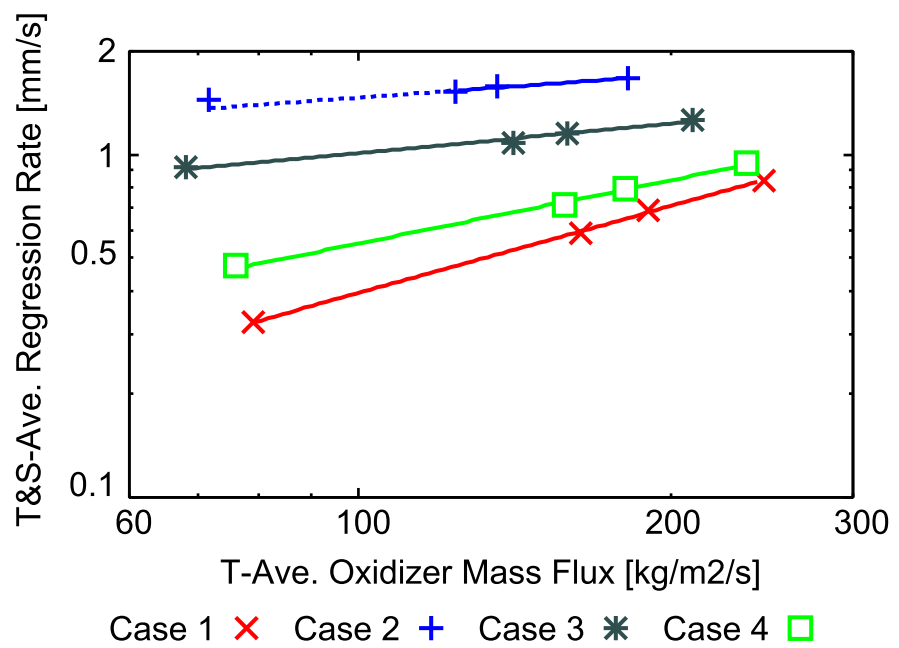

Fig. 9 Time- and space-averaged regression rates are estimated with the model which includes radiative heat transfer. The gas emissivity $\varepsilon_{g}$ is changed into various values. The interpolated lines are the fitted lines to the empirical expression $\bar{r}=a \bar{G}_{o x}^{n}$. However, Test No.3 (lowest mass-flux point) in Case 2 is not used by fitting the expression owing to poor convergence of the numerical solution. The $x$-axis and the $y$-axis are log scale.

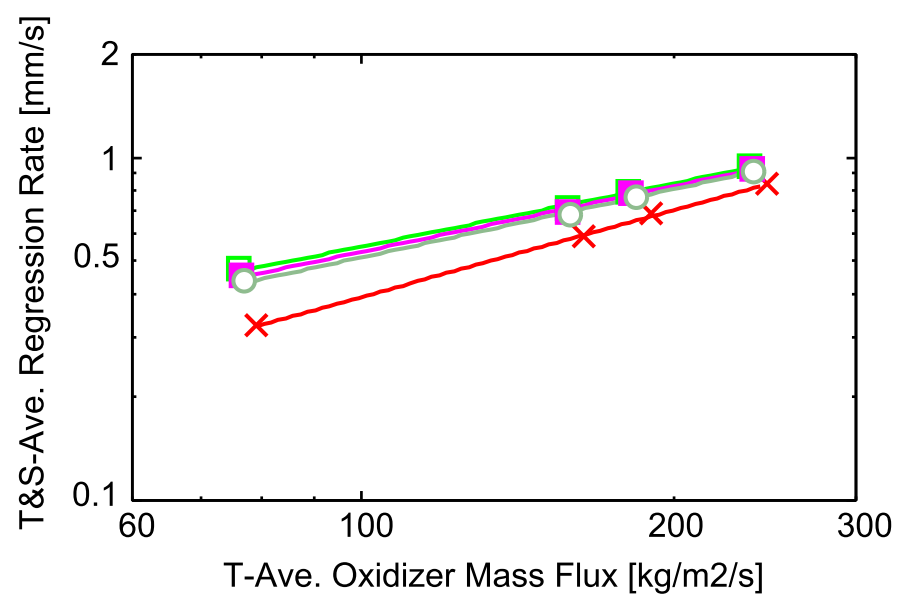

Case $1 \times \quad$ Case $4 \square \quad$ Case $5 \square \quad$ Case 6

Fig. 10 Time- and space-averaged regression rates are estimated with the model which includes radiative heat transfer. The solid fuel emissivity $\varepsilon_{s}$ is changed into various values. The interpolated lines are the fitted lines to the empirical expression $\bar{r}=a \bar{G}_{o x}^{n}$. The $x$-axis and the $y$-axis are log scale. 
understood in more detail. This kind of study is planned for next works.

\subsection{Calculation Cost}

In order to evaluate calculation cost, computational time is measured. The computer is DELL Precision ${ }^{\mathrm{TM}}$ T7400. The CPU is Intel ${ }^{\circledR}$ Xeon ${ }^{\circledR}$ CPU X5482, $3.20 \mathrm{GHz}, 4$ cores. The compiler is Intel ${ }^{\circledR}$ Fortran Compiler XE 12.0 Update 5 for Linux. The automaticoptimization option (-fast) and the automatic-parallelization option (-parallel) are used when the source codes are compiled. Each test in numerical simulations involves 11 convergent results corresponding to time $t$. Typical test has been selected as the representative case for the calculation time measurement. In this simulation, the initial condition at $t=0$ [s] is the uniform oxidizer flowfield. As a result, for calculating one convergent result, the wall clock time is about 9 minutes, while the CPU time is about 31 minutes.

\section{Concluding Remarks}

We developed an estimation method for hybrid rocket internal ballistics. In order to validate the developed method, the calculation results were compared with the experimental data. While the numerical estimation of time- and space-averaged regression rates is of the same order of magnitude as the experimental data, the factor $n$ for the calculations differs from that obtained by experiments. The main reason is that only convective heat transfer is considered as the form of heat feedback from flame. It has been demonstrated that the model with radiation allows to improve the prediction accuracy of the regression rate dependency on oxidizer mass flux.

As future works, in order to evaluate and to validate the method with a higher accuracy, a comparison using more experimental data is required. And, in order to obtain a better comprehension of the radiation effects, an estimation model for the radiation parameters has to be implemented.

\section{Acknowledgment}

This research is supported by the Hybrid Rocket Research Working Group (HRrWG) of Institute of Space and Astronautical Science, Japan Aerospace Exploration Agency. The authors thank members of HRrWG for their helpful discussion.

\section{References}

( 1 ) Antoniou, A. and Akyuzulu, M., A Physics Based Comprehensive Mathematical Model to Predict Motor Performance in Hybrid Rocket Propulsion System, Proceedings of the 41st AIAA/ASME/SAE/ASEE Joint Propulsion Conference $\mathcal{F}$ Exhibit, AIAA20053541(2005-7), pp.1-16.

( 2 ) Stoia-Djeska, M. and Mingireanu, F., A Computational Fluid Dynamics Based Stability Analysis for Hybrid Rocket Motor Combustion, Proceedings of the 16th AIAA/CEAS Aeroacoustics Conference, AIAA2010-3909(2010-6), pp.1-10.

( 3 ) Funami, Y. and Shimada, T., Hybrid Rocket Performance Prediction with Coupling Method of CFD and Thermal Conduction Calculation, Transactions of the Japan Society for Aeronautical and Space Sciences, Aerospace Technology Japan, Vol.10, No.ists28 (2012), pp.Pa_71-Pa_76, (http://www . jstage.jst.go.jp/browse/tastj).

( 4 ) Gordon, S. and McBride, B.J., Computer Program for Calculation of Complex Chemical Equilibrium Compositions and Applications, NASA Reference Publication, 1311 (1994), pp.1-55.

( 5 ) Karabeyglu, A., Combustion Instability and Transient Behavior in Hybrid Rocket Motors, Fundamentals of Hybrid Rocket Combustion and Propulsion, (2007), pp.351411, American Institute of Aeronautics and Astronautics.

( 6 ) Marxman, G.A., Combustion in the Turbulent Boundary Layer on a Vaporizing Surface, Proceedings of the 10th Symposium (International) on Combustion, (1964-8), pp.13371349. 
( 7 ) Marxman, G.A., Wooldridge, C.E. and Muzzy, R.J., Fundamentals of Hybrid Boundary Layer Combustion, Proceedings of the Heterogeneous Combustion Conference, AIAA63-505(1963-12), pp.1-17.

( 8 ) Lengelle, G., Solid-Fuel Pyrolysis Phenomena and Regression Rate, Part 1: Mechanisms, Fundamentals of Hybrid Rocket Combustion and Propulsion, (2007), pp.127-165, American Institute of Aeronautics and Astronautics.

( 9 ) Wada, Y. and Liou, M.-S., A Flux Splitting Scheme With High-Resolution and Robustness for Discontinuities, NASA Technical Memorandum, 106452 (1994), pp.123.

(10) Karabeyglu, M.A., Cantwell, B.J. and Stevens, J., Evaluation of Homologous Series of Normal-Alkanes as Hybrid Rocket Fuels, Proceedings of the 41st AIAA/ASME/SAE/ASEE Joint Propulsion Conference E Exhibit, AIAA2005-3908(20057), pp.1-42.

(11) Karabeyglu, M.A., Cantwell, B.J. and Zilliac, G., Development of Scalable Space-Time Averaged Regression Rate Expressions for Hybrid Rockets, Journal of Propulsion and Power, Vol.23, No.4(2007), pp.737-747. 